\title{
Recent progress at the Keck Interferometer
}

S. Ragland, R. Akeson, M. Colavita, R. Millan-Gabet, J. Woillez, et al.

S. Ragland, R. Akeson, M. Colavita, R. Millan-Gabet, J. Woillez, P. Wizinowich, E. Appleby, B. Berkey, A. Cooper, C. Felizardo, J. Herstein, M. Hrynevych, D. Medeiros, D. Morrison, T. Panteleeva, J.-U. Pott, B. Smith, K. Summers, K. Tsubota, C. Tyau, E. Wetherell, "Recent progress at the Keck Interferometer," Proc. SPIE 7734, Optical and Infrared Interferometry II, 773402 (16 July 2010); doi: 10.1117/12.857477

Event: SPIE Astronomical Telescopes + Instrumentation, 2010, San Diego, California, United States 


\title{
Recent progress at the Keck Interferometer
}

\author{
S. Ragland, ${ }^{\text {a }}$ R. Akeson, ${ }^{\mathrm{b}}$ M. Colavita, ${ }^{\mathrm{c}}$ R. Millan-Gabet, ${ }^{\mathrm{b}}$ J. Woillez, ${ }^{\mathrm{a}}$ P. Wizinowich, ${ }^{\mathrm{a}}$ E. Appleby, ${ }^{\mathrm{a}}$ \\ B. Berkey, ${ }^{a}$ A. Cooper, ${ }^{a}$ C. Felizardo, ${ }^{b}$ J. Herstein, ${ }^{b}$ \\ M. Hrynevych, ${ }^{a}$ D. Medeiros, ${ }^{a}$ D. Morrison, ${ }^{a}$ T. Panteleeva, ${ }^{a}$ J.-U. Pott, ${ }^{a}$ B. Smith, ${ }^{a}$ K. Summers, ${ }^{a}$ K. \\ Tsubota, ${ }^{\mathrm{a}}$ C. Tyau, ${ }^{\mathrm{a}}$ E. Wetherell ${ }^{\mathrm{a}}$
}

\author{
${ }^{a}$ W. M. Keck Observatory, 65-1120 Mamalahoa Hwy, Kamuela, HI 96743 \\ bASA Exoplanet Science Institute, California Institute of Technology, \\ 100-22, Pasadena, CA 91125 \\ ${ }^{\mathrm{c}}$ Jet Propulsion Laboratory, California Institute of Technology, \\ 4800 Oak Grove Dr., Pasadena, CA 91109
}

\begin{abstract}
The Keck Interferometer (KI) combines the two $10 \mathrm{~m}$ diameter Keck telescopes providing milliarcsecond angular resolution. KI has unique observing capabilities such as sensitive $\mathrm{K}$-band $\mathrm{V}^{2}$, L-band $\mathrm{V}^{2}$ and $\mathrm{N}$-band nulling operations. The instrument status of the Keck Interferometer since the last SPIE meeting in 2008 is summarized. We discuss the performance of new visibility observing capabilities including L-band and self-phase referencing modes. A simultaneous dual-beam-combiner mode in the $\mathrm{K}$ and L-band has been demonstrated, nearly doubling operational efficiency for bright targets. Operational improvements including simplified reliable operations with reduced personnel resources are highlighted. We conclude with a brief review of the current and future developmental activities of KI. Details of ASTRA developments, nulling performance and science results are presented elsewhere at this conference.
\end{abstract}

Keywords: Interferometry, Visibility-squared, Nulling, L-band interferometry

\section{INTRODUCTION}

Keck Interferometer (KI) has been operational since 2001, with shared risk science starting in June 2002, and formal commissioning of the first V2 modes in 2004. The progress of the KI is presented in the several previous SPIE papers. ${ }^{1,2,3,4,5}$. The priorities of KI during the past two years have been completion of Nuller key science observations, ${ }^{6,7}$ commissioning of Self-Phase Referencing (SPR) and Dual-Field Phase Referencing (DPFR) modes of the ASTRA project, $^{8}$ and carrying out of TAC allocated science observations ${ }^{9}$ in various operational modes. In addition, several infrastructure and operational improvements have been made during this period in order to improve the observing efficiency and add new observing capabilities. In this paper, we discuss the progress at the Keck Interferometer in terms of operational capabilities, and infrastructure and operational improvements, with special emphasis on what was implemented since the last SPIE meeting in Marseille, 2008.

The KI is a NASA funded project to perform long baseline interferometry with the two $10 \mathrm{~m}$ diameter Keck telescopes in the near and mid-infrared wavelengths. The physical baseline (B) of KI is $85 \mathrm{~m}$ providing an angular resolution $(\lambda / 2 \mathrm{~B})$ of $\sim 3$ mas at $2.2 \mu \mathrm{m} \& 12$ mas at $10 \mu \mathrm{m}$.

Since the 2008 SPIE meeting, KI operated on the sky for 66 nights - an average of $\sim 33$ nights per year. Of these, 23 nights were for V2 science, 9.5 nights for V2 engineering (including ASTRA engineering), 29 nights for Nuller science and 4.5 nights ( 9 half-nights) for Nuller engineering. We continued to improve the observing efficiency of KI during this 2-year period through operations automation, preventive maintenance of the interferometer subsystems, staff training and appropriate policy changes. KI is currently used for a variety of astrophysical studies. There were 8 refereed publications during this period, including 6 papers on V2 observations and 2 papers on Nuller observations.

Optical and Infrared Interferometry II, edited by William C. Danchi, Françoise Delplancke, Jayadev K. Rajagopal, Proc. of SPIE Vol. 7734,773402 - (c) 2010 SPIE · CCC code: 0277-786X/10/\$18 · doi: 10.1117/12.857477 
In this paper, we describe the various operational capabilities of KI with emphasis on recently implemented modes in Section 2, recent operational improvements in Section 3, highlights of science operations in Section 4, and a brief description of the plans for near future developments in Section 5.

\section{CURRENT CAPABILITIES}

The Keck Interferometer (KI) has unique operational capabilities such as sensitive $\mathrm{V}^{2}$ measurements in the K-band, $\mathrm{V}^{2}$ measurements in the L-band, and nulling measurements in the N-band. Other operational capabilities include H-band $\mathrm{V}^{2}$ and high spectral resolution measurements through self-phase referencing $\left(\mathrm{V}^{2}\right.$-SPR) and simultaneous $\mathrm{V}^{2}$ measurements in the $\mathrm{K}$ and $\mathrm{L}$ bands $\left(\mathrm{V}^{2}-\mathrm{K} / \mathrm{L}\right.$ mode), and more recently, dual field phase referencing ( $\mathrm{V}^{2}$-DFPR) capability. A compete list of all available operational modes of the KI is given in Table 1 along with the current performance. A schematic diagram of the beam-combiner and fast delay line laboratories is shown in Figure 1. New beam-combiners and infrared cameras were integrated to accommodate new operational capabilities. More recently, we opted to use the ASTRA configuration for beam extraction from the telescope for all observing modes except for the nuller and $\mathrm{V}^{2}-\mathrm{K} / \mathrm{L}$ modes. This enables simplified scheduling of different $\mathrm{V}^{2}$ modes, and avoids frequent reconfigurations of the dual star module. Two relatively recent capabilities to improve spectral resolution and spectral coverage are described in Sections $2.1 \&$ 2.2 in some detail.

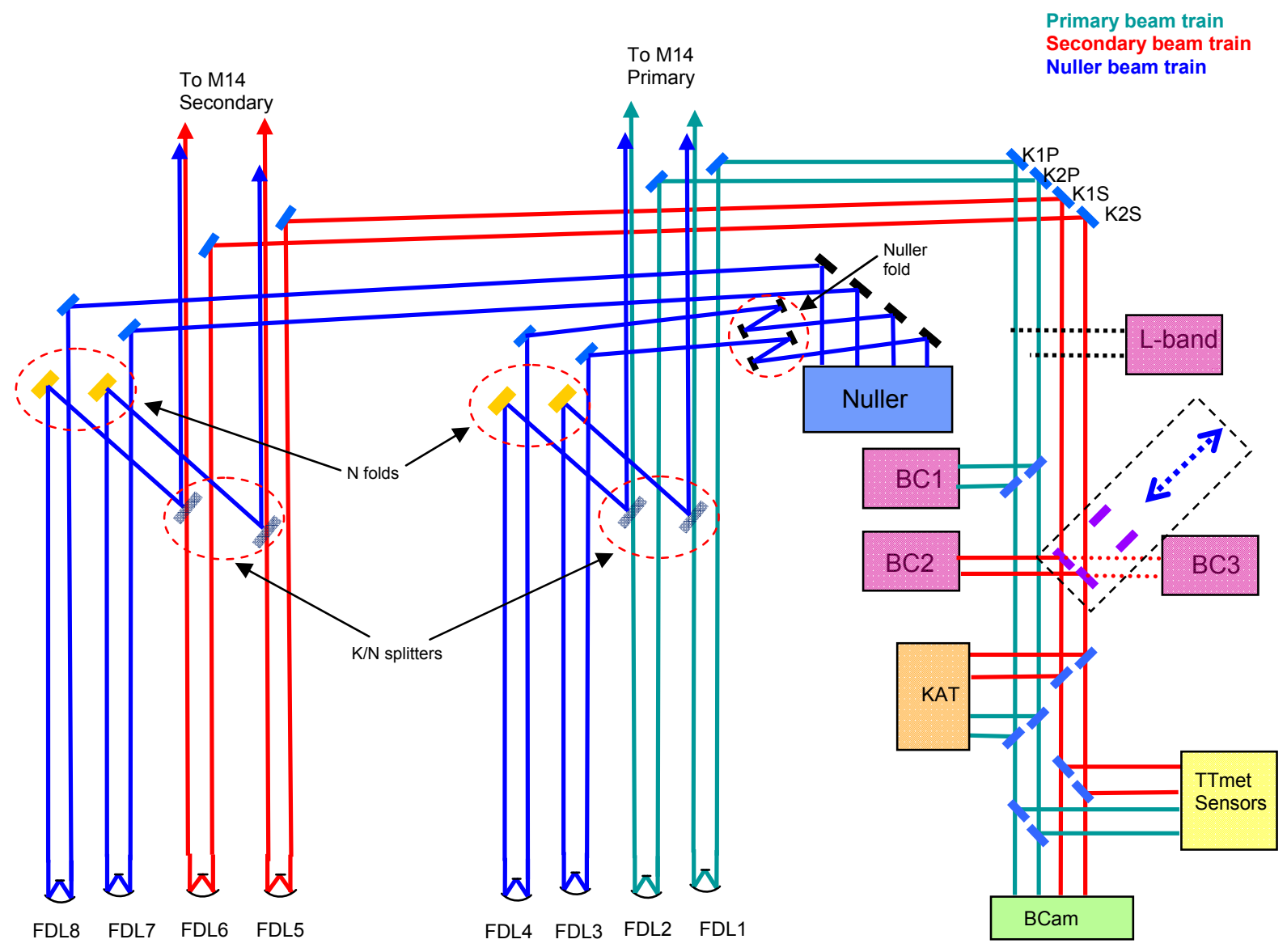

Figure 1: Schematic layout of the beam-combiner and the fast delay line laboratories. Standard K-band and Hband $\mathrm{V}^{2}$ instruments use beam-combiner 1 (BC1), the Nuller instrument uses the Nuller beam-combiner and beam-combiners $1 \& 2$ (BC1 \& BC2), the L-band mode uses the L-band beam-combiner, and the ASTRA modes use beam-combiners $1 \& 3$ (BC1 \& BC3). All these modes use the angle tracker (KAT) and the alignment camera, BCAM. 


\begin{tabular}{|l|l|l|}
\hline & KI capabilities & Current Performance \\
\hline 1 & Nulling Mode & N-band flux $>1.7$ Jy \\
\hline 2 & $\mathrm{~V}^{2}-\mathrm{K} 5 ; \mathrm{R} \sim 27$ & $\mathrm{~K}^{\prime}<10.3$ \\
\hline 3 & $\mathrm{~V}^{2}-\mathrm{K} 10 ; \mathrm{R} \sim 54$ & $\mathrm{~K}^{\prime}<9.5$ \\
\hline 4 & $\mathrm{~V}^{2}-\mathrm{K} 42 ; \mathrm{R} \sim 230$ & $\mathrm{~K}^{\prime}<7.6$ \\
\hline 5 & $\mathrm{~V}^{2}-\mathrm{SPR} ; \mathrm{R} \sim 2000$ & $\mathrm{~K}^{\prime}<7$ \\
\hline 6 & $\mathrm{~V}^{2}-\mathrm{H} 4 ; \mathrm{R} \sim 22$ & $\mathrm{H}<9$ \\
\hline 7 & $\mathrm{~V}^{2}-\mathrm{L} 10 ; \mathrm{R} \sim 63$ & $\mathrm{~L}^{\prime}<6$ \\
\hline 8 & $\mathrm{~V}^{2}-\mathrm{K} / \mathrm{L}$ & $\mathrm{K}$ \\
\hline 9 & $\mathrm{~V}^{2}-\mathrm{DFPR}$ & $\mathrm{K}<12.7$ \& L' $<4.8$ \\
\hline
\end{tabular}

Table 1: Operational capabilities of the Keck Interferometer

\subsection{Self-phase Referencing (SPR) Mode}

The SPR mode is part of the ASTRA project ${ }^{8}$ funded by NSF. In this mode, part of the K-band light is passed to a standalone K-band fringe tracker and the remaining light is passed to a science beam-combiner (Figure 2). The fringe tracker, operating at 200 or $250 \mathrm{~Hz}$, stabilizes the science fringes scanned at a slow rate of $\sim 1 \mathrm{~Hz}$. The SPR mode enables high spectral resolution observations of spectral resolution with an $\mathrm{R}(=\lambda / \delta \lambda)$ of 2000 (which is Nyquist sampled at $\mathrm{R} \sim$ 1000; 330 spectral channels) for targets as faint as $\mathrm{K} \sim 7$. This mode has recently been made available as a standard observing mode. ${ }^{10,11,12,13}$ The schematic diagram of this new operational mode is given in Figure 2.

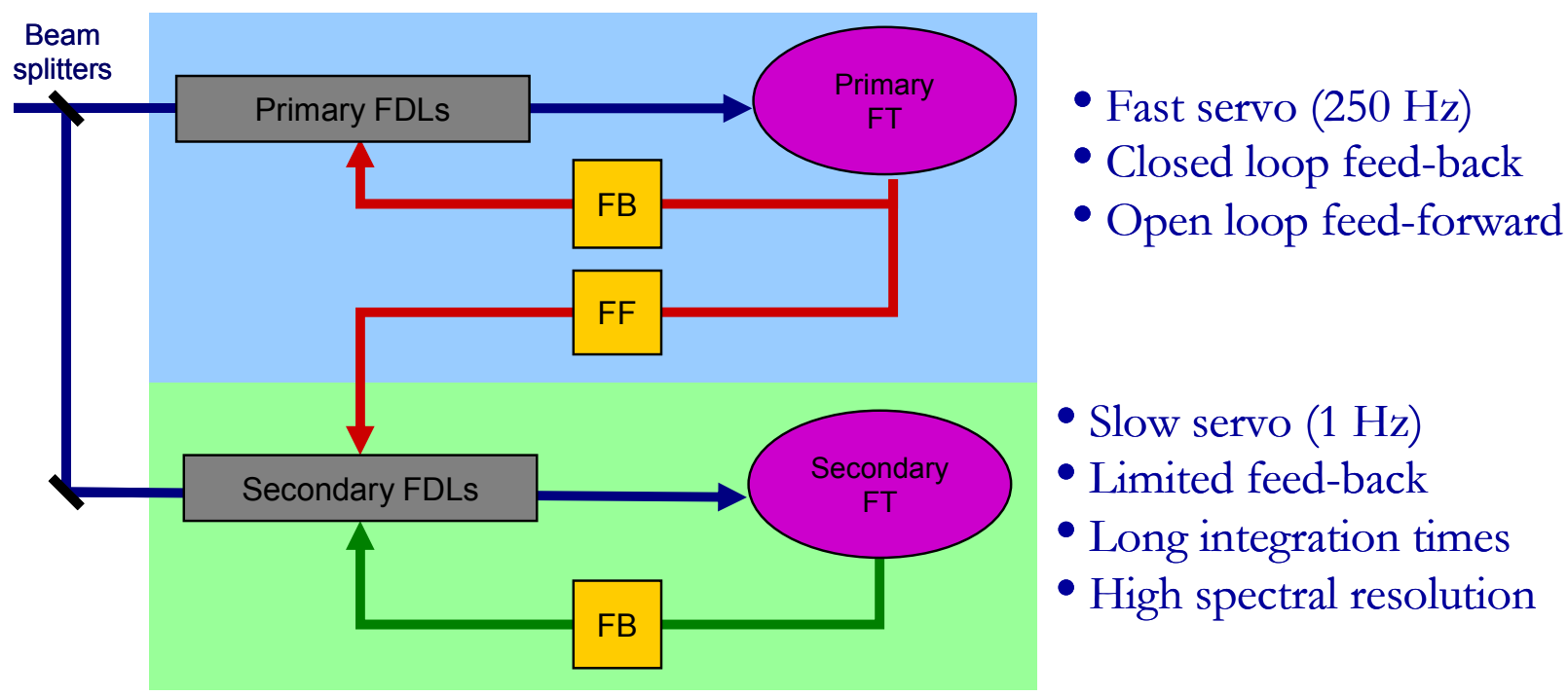

Figure 2: Schematic diagram of SPR instrument.

The SPR capability also provides the opportunity to exploit the power of new observables, namely differential (with respect to other spectral channels) visibility amplitudes and phases, which can often be calibrated to greater precision than their absolute levels. Visibility amplitudes for each spectral channel were always a standard KI data product. We now also compute and output the phase for each spectral channel, referenced to the white light phase. The differential phases are now also part of the standard quick-look plot products of both the near-real time and end-of-night pipelines. In addition, we now also produce quick-look plots versus wavelength of all the other quantities of interest (visibility, flux, telescope flux ratios).

We also implemented an improved wavelength calibration for the SPR mode. A new stage with a Neon lamp was installed in one beam path of the secondary camera. The use of this lamp enables alignment of the high dispersion grism spectral channel to a one pixel tolerance, corresponding to a wavelength calibration for the SPR mode of less than $1 \mathrm{~nm}$. 


\subsection{L-band and Simultaneous K/L Mode}

The L-band $\mathrm{V}^{2}$ instrument uses the L-band beam-combiner equipped with a PICNIC camera. In addition, a simultaneous $\mathrm{K}$ and $\mathrm{L}$ band observing mode was implemented by splitting the telescope pupils into two halves and sending one half to the K-band instrument and the other half to the L-band instrument. This L-band instrument is currently available for shared-risk science, and first science results from this instrument were published last year. ${ }^{14}$ More recently, the observing scheme for L-band mode has been optimized and an observing sequencer was developed. Final validation of this new operational tool is planned for summer 2010.

\section{OPERATIONAL IMPROVEMENTS}

\subsection{New/Upgraded Operational Tools}

We have developed or improved several operational tools during the last two years. These new tools continue to improve our operational efficiency. Basic operational tools such as "IFoper" and "IFsys" underwent upgrades because of the addition of new subsystems, motors, Picomotors, and shutters as a part of instrument development. The FATCAT camera initialization tool applies fringe scan parameters to appropriate fast delay lines. In the following sections, we briefly discuss a few significant improvements.

\subsubsection{Auto Alignment Improvements}

Beam train alignment from the telescope (AO enclosure) to the science cameras in the basement ( $\sim 150 \mathrm{~m}$ path) is one of the critical elements of day time preparation of the interferometer for observations. Most complicated and time consuming alignments are automated, reducing manpower and human error, and during the past two years, we continued to improve these auto-alignment (AA) scripts and alignment procedures.

Six separate scripts that existed for V2/Nuller beam alignments were combined into two general scripts: "Align_Primary" and "Align_Secondary". A screen shot of the secondary beam AutoAlignment GUI is shown in Figure 3. The very first step of the AA tool is to run "Configure script" which enables the user to select the KAT (angle tracker) internal filter ( $\mathrm{J}$ or H), telescope ID (Keck 1 or Keck 2), and operational mode (V2, Nuller or ASTRA). This configuration option is available during a few following steps to allow the operator to reconfigure the input parameters if needed.

A new setup procedure was developed to prepare the beam train for alignment. This procedure opens all shutters and hatches in the beam path, closes all sources not used for alignment (WL, cameras and metrology lasers, LEDs). Depending on the operational mode and telescope ID, appropriate optical stages in the basement, coude train and the $\mathrm{AO}$ bench are moved to the desired position. Some alignment procedures were modified to limit software errors or to improve AA performance. As an example, the fields-of-view of a few cameras were reduced to reject ghost images, and motor move methods were modified to have better repeatability.

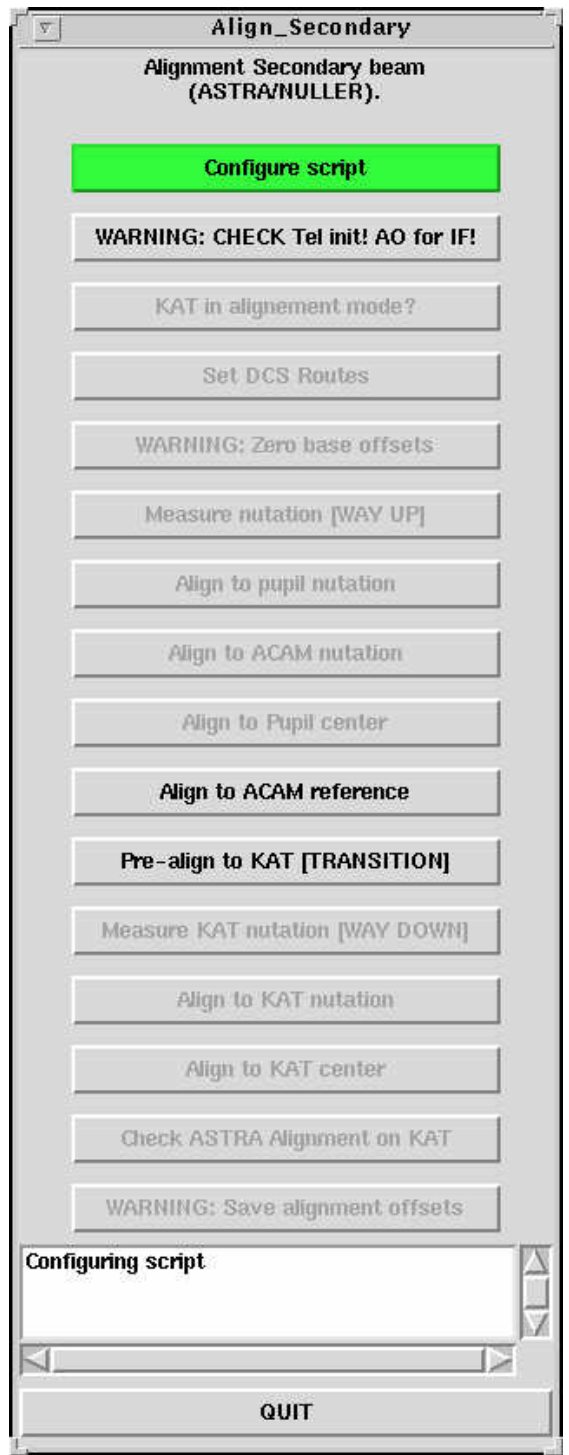

Figure 3: A screen-shot of an AA GUI. 


\subsubsection{SMC-based Sequencers}

The most significant software upgrade to the KI over the last two years has been the transition from a Rhapsody-based V2 sequencer to a State Machine Compiler (SMC)-based sequencer. The Nuller Sequencer (detailed in the 2008 SPIE paper $^{4}$ ) was the first of the Interferometer sequencers to be based on the State Machine Compiler. It was written from scratch to support Nuller operations.

The limitations of the original $\mathrm{V}^{2}$ sequencer, combined with the robustness of the Nuller Sequencer and the need for sequencers to support new modes, drove us to develop a V2 sequencer using the SMC architecture used in the Nuller Sequencer implementation.

The Interferometer Sequencer (ISEQ) infrastructure is a Solaris CORBA application using the JPL real-time core (RTC) software. ISEQ uses RTC to access the configuration database (PostgreSQL) for initialization, and to publish and subscribe to the real-time telemetry. The ISEQ code is primarily written in $\mathrm{C}++$, with Python used for the user interfaces. Additionally, a Target Manager tool was created as part of the SMC sequencer infrastructure. The TargetManager is a Python CORBA application that allows an operator to select a star from the catalog file and provide the selected target information to any of the sequencers. The TargetManager is used by all of the ISEQ top-level sequencers. A screen-shot of TargetManager tool is shown in Figure 4.

\begin{tabular}{|c|c|c|c|c|c|c|c|c|c|c|c|c|}
\hline 5 & & & & Targe & t Man & lager - D & ault & & & & & \\
\hline \multicolumn{13}{|l|}{ File } \\
\hline Name & RAh & RAm & RAs & $\mathrm{DECd}$ & DECm & $\mathrm{DECs}$ & RA-Mot & DEC-Mot & VBand & $\mathrm{KB}$ and & BVColor & \\
\hline $\mathrm{HD} 167654$ & 18 & 16 & 0.5 .572 & +02 & 22 & 39.060 & -0.007 & -0.029 & 6.1 & 1.0 & 1.55 & $\Delta$ \\
\hline HD 159561 & 17 & 34 & 56.071 & +12 & 33 & 36.125 & 0.113 & -0.223 & 2.1 & 1.7 & 0.15 & \\
\hline $\mathrm{HD} 171443$ & 18 & 35 & 12.427 & -08 & 14 & 38.662 & -0.019 & -0.315 & 3.9 & 0.8 & 1.32 & \\
\hline $\mathrm{HD} 169414$ & 18 & 23 & 41.890 & +21 & 46 & 11.107 & 0.211 & -0.243 & 3.9 & 1.3 & 1.17 & \\
\hline MUTC_325 & 20 & 03 & 02.510 & +05 & 44 & 16.676 & 0.001 & -0.008 & 7.8 & 5.9 & 0.11 & \\
\hline $\mathrm{HD} 188107$ & 19 & 53 & 22.605 & +04 & 24 & 01.413 & 0.010 & -0.000 & 6.5 & 6.6 & 0.01 & \\
\hline HD 188385 & 19 & 54 & 40.200 & +07 & 08 & 25.271 & 0.029 & -0.000 & 6.1 & 6.1 & 0.04 & \\
\hline HD 190007 & 20 & 02 & 47.045 & +03 & 19 & 34.278 & -0.092 & 0.121 & 7.5 & 4.8 & 1.13 & \\
\hline $\mathrm{HD} 192343$ & 20 & 14 & 09.179 & +06 & 34 & 37.955 & -0.131 & -0.062 & 8.0 & 6.5 & 0.68 & \\
\hline $\mathrm{HD} 187691$ & 19 & 51 & 01.643 & +10 & 24 & 56.617 & 0.244 & -0.135 & 5.1 & 3.9 & 0.56 & \\
\hline \multirow{2}{*}{\multicolumn{12}{|c|}{$\begin{array}{l}\text { Catalog: /archivedisk/archive/schedules/tst.cat } \\
\text { Target: Target Not selected }\end{array}$}} & \\
\hline & & & & & & & & & & & & \\
\hline & \multicolumn{3}{|c|}{ Load Catalog } & \multicolumn{3}{|c|}{ Set Target } & \multicolumn{2}{|c|}{ Refresh } & & & & \\
\hline
\end{tabular}

Figure 4: A screen-shot of Target Manager

Using a different design from the Nuller Sequencer, separate ISEQ sequencers were designed to encapsulate one or more RTC gizmos (or other subsystems, i.e. AO) instantiations so that these sequencers could be re-used by higher-level sequencers for different modes. We currently have or are developing the following high-level sequencers:

- V2 mode which controls a single fringe tracker, two fast delay lines, and two angle tracker gizmos (Figure 4)

- SPR mode which controls two $\mathrm{V}^{2}$ sequencers (each controlling the above gizmos)

- L-band mode which controls the same generic gizmos as $\mathrm{V}^{2}$ mode, but which uses a different sequence

- $\mathrm{L}+\mathrm{K}$ mode which controls four fast delay lines and four angle tracker gizmos

- DFPR mode will use an Observing Sequencer that will control two V2 sequencers and two acquisition sequencers (controlling both telescopes and $\mathrm{AO}$ systems)

High-level sequencers communicate with lower-level sequencers (subsequencers), and sequencers communicate with RTC gizmos using CORBA IDL methods. Gizmos respond to sequencers and subsequencers respond to higher-level sequencers by sending telemetry. The important telemetry from a subsequencer to a higher-level sequencer is usually the state. The higher-level sequencer typically transitions when it gets the right state telemetry from the subsequencer. 


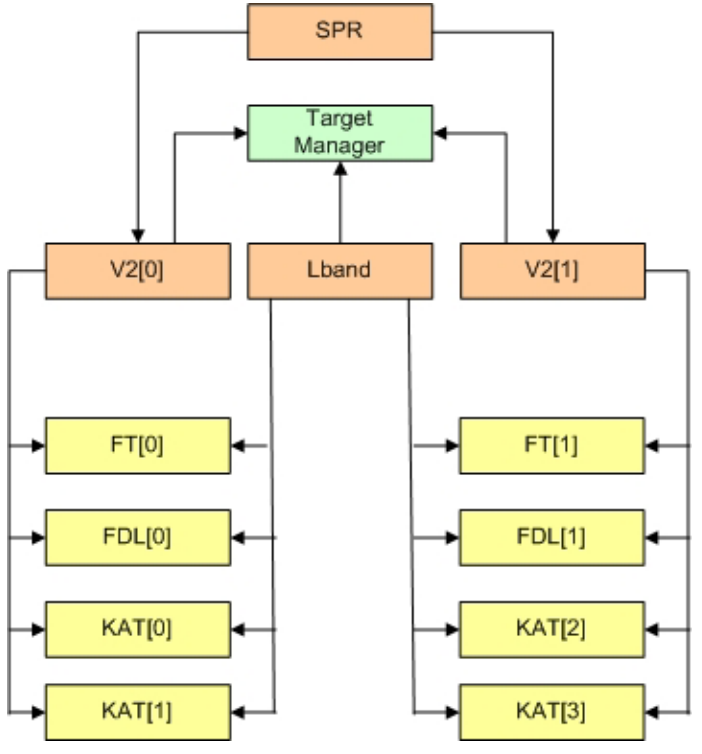

Figure 5: Collaboration Diagram of ISEQ Sequencers

Each of the top-level sequencer GUIs is similar, providing: the currently selected target information, the status of each of the subsequencers, the baseline information, LDL position, and control buttons for the sequence.

SPR and V2 versions of the sequencers have both been recently sky-tested and are used regularly for science. The Lband, $\mathrm{L}+\mathrm{K}$, and Observing Sequencer versions are planned to be sky-tested in the coming months.

\subsubsection{FTS Scan Improvement}

The spectral calibration of the fringe-tracker and science cameras are performed through FTS scans. Recently, two scripts were developed to partially automate the FTS scans used for wavelength calibration. Although the setup for FTS scans and acquiring fringes involves manual procedures, the new script launches the data archive application, performs the dark calibration and records the specified amount of fringe data. Another script processes the archived data and produces a plot, a wavelength file and waveform config file in the format readable by the camera software. This new spectral channel wavelength calibration improves accuracy and reduces human error.

\subsubsection{Alarm Handler Tool}

A new tool, namely, "Alarm Handler" was developed to display IF Motion Control system status. This tool is launched from the IFoper/Resource menu. An indicator associated with each PMAC unit changes its color to Red or White when a PMAC faults and sounds an audible alarm. Essentially, one could run this tool in the background and hear an alarm if any of the PMAC devices faults. The "Alarm Handler" also shows the affected devices on the specific PMAC and displays the recovery procedure. A screen-shot of Alarm Handler tool is shown in Figure 6. 


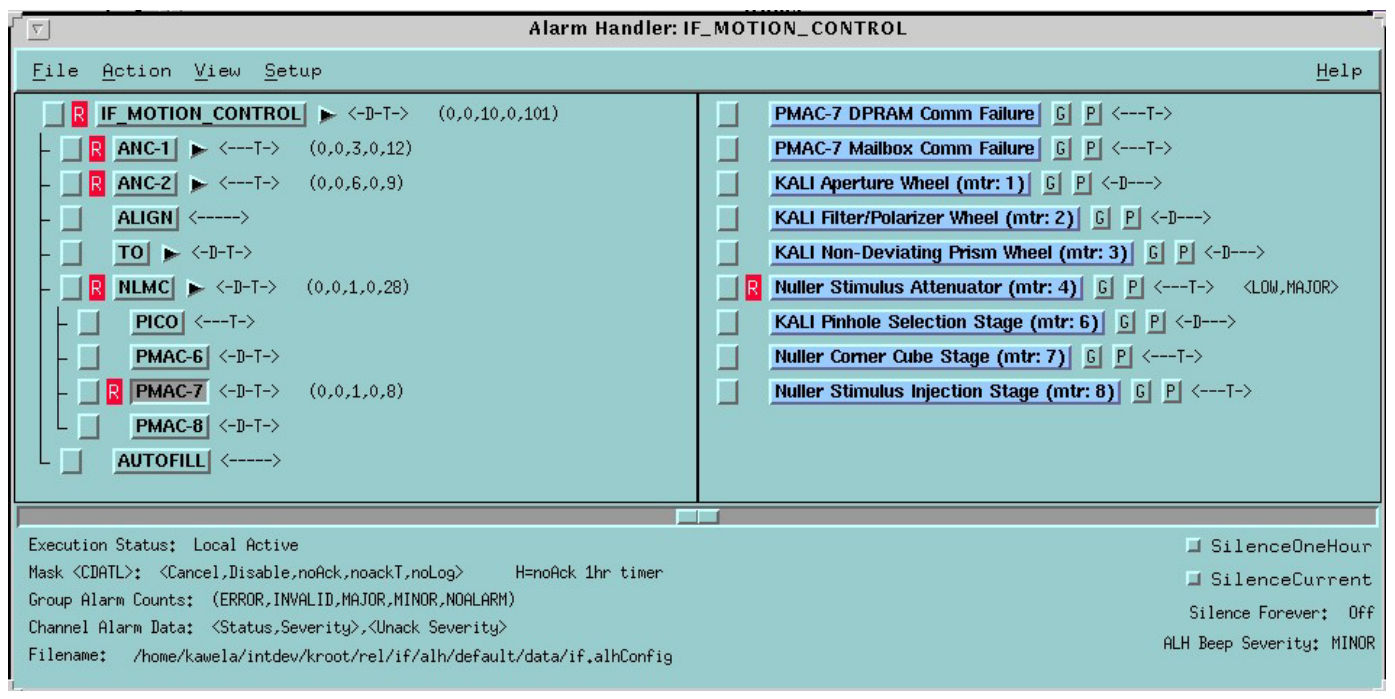

Figure 6: A screenshot of the Alarm Handler GUI

\subsection{Procedural Improvements}

There were several procedural changes put in place during the last two years in preparing and operating the interferometer. The changes include internal focus optimization of FATCAT camera (when the camera is warm) and whitelight to boresight laser co-alignment of the L-band instrument.

New procedures for reconfiguration between various modes were developed as Interferometer operational capabilities were increased. These procedures include $\mathrm{V}^{2}$ to SPR mode or ASTRA to Nuller configuration procedure, SPR specific beam-train alignment preparations, etc.

We continue to use week-before-run, day-before-run and day-of-run checklists to avoid human error. These checklists also provide historical information on alignment and camera performance, as a number of values (flux, noise, etc.) are entered on the checklist. This document continues to be updated as the preparation procedures evolve and obscure failure modes are identified. Recently, we combined individual document for various observing mode into a single more general checklist document. Excel Visual Basic package was applied to switch between IF modes or Summit and Head quarter tasks. It saves time to implement any new changes or additions to check list.

Identifying maintenance tasks and performance tests is another ongoing area of improvement. The maintenance tasks performed during the last two years include snowing of mirrors, removing residual shear on the coude metrology source plate, rotating $\mathrm{H}+\mathrm{K}$ dichroics so that their wedge angles were the same for both beams in order to reduce aberration on the angle tracking camera, replacing diode lasers used for the boresight source, peaking up the metrology signal by adjusting metrology source plate optics, maintaining Picomotors, finding the center of nominal travel for ASTRA/Nuller mirror positions, realigning FDL metrology, inspecting coude optics and mirrors, and checking the focus of FDLs and beam-combiners. Maintenance tasks also include inspection/lubrication of motion stages, replacement of optics, and vacuum service on cryostats, delay line track inspection / cleaning, and oxygen alarm calibrations. We also performed periodic tests including an overnight test to analyze the Fast Delay Line jitter performance and power spectrum analysis of the accelerometer data to check the health of these subsystems and scheduled necessary maintenance work. Both of these tests have caught degraded performance that would have impacted observing. Maintaining a stock of critical spares is an ongoing effort to keep a system of this complexity functioning. So far, we have been successful in preventing a significant loss of observing time due to lack of spare parts.

The dichroics feeding the fringe trackers received actuated translation stages to ease reconfiguration between two sets of dichroics; one set optimized for H-band angle tracking and the other set optimized for H-band fringe tracking. Actuation of this stage enables easy night time observing mode changes. A custom multiplexer box used with New Focus 
Picomotors was modified to accommodate 16 motors, instead of the previous 12 , helping to consolidate control and allow expansion for SPR/DFPR modes.

The configuration control process at the $\mathrm{KI}$ is working well in protecting the operational system, while at the same time enabling development of new operational capabilities and upgrades. The configuration control board (CCB) reviews the engineering change requests (ECR) and field change notices (FCN). There were 299 new ECR/FCNs, 203 closed, and 30 cancelled since 1 July 2008 (Software: New: 251, Closed: 160, Cancelled: 29; Electronics: New: 27, Closed: 24; Cancelled: none; Mechanical: New: 16, Closed: 14, Cancelled: 1; IF operations: New: 5, Closed: 5, Cancelled: none). We also reopened 2 software and 4 mechanical ECRs during this two year period. All modified IF subsystems are captured and frozen in-situ then rebuilt from scratch on a separate host after every run.

\subsection{Upgrades to the Infrastructure for ASTRA}

Under the ASTRA project many upgrades to the hardware, real-time software and tools infrastructure were implemented over the last two years. The most interesting are:

- Upgrades to the real-time core (RTC) and the Keck RTC (KRTC) for slower than $1 \mathrm{~Hz}$ camera frame rates to support longer fringe tracker integration times

- Upgrades to the fringe tracker software and tools to speed up the initialization process to allow clocks to be changed at run time.

\subsection{Cryogenic Camera Autofill}

The liquid nitrogen used to cool the various interferometric detectors is supplied by an automatic fill system. While this has saved a great deal of labor in avoiding manual fills, the system requires a moderate amount of effort to maintain. Simple in theory, in practice the autofill system has proven to be a very dynamic system. Variations in load conditions, supply pressures and the number of active camera dewars conspire to create faults that result in either wasted cryogen or incomplete fills.

System automation is implemented with a programmable logic controller (PLC). While simple and reliable, creating a PLC code that adequately responds to the varying conditions has proven challenging.

Some of the issues stem from the variations in the liquid nitrogen supply dewars. The dewars, supplied by a local vendor, vary in models and features, as well as condition. These differences vary the rate at which gas pressure is generated and is available to feed nitrogen into the supply lines.

To improve the performance of the autofill system we are moving to increased complexity in the PLC code and the addition of sensors to allow better monitoring. Temperature sensors throughout the system allow the detection of liquid nitrogen at each critical point in the path, but there is currently no remote way of reading the supply pressure or allowing the PLC to respond to pressure events. Addition of an electronic pressure gauge at the gas supply dewar is planned. Implementation of the pressure gauge and associated control logic in the PLC should improve reliability.

\subsection{Camera Cooling Challenges}

After numerous thermal cycles, the FATCAT-S focal plane array (FPA) began to exhibit a slow thermal response and would not achieve its nominal cold operating temperature of $\sim 76 \mathrm{~K}$. The nitrogen cold plate remained at $\sim 84 \mathrm{~K}$. The trend is illustrated in Figure 7. An increase in the thermal impedance between the cold plate and the detector was suspected. 


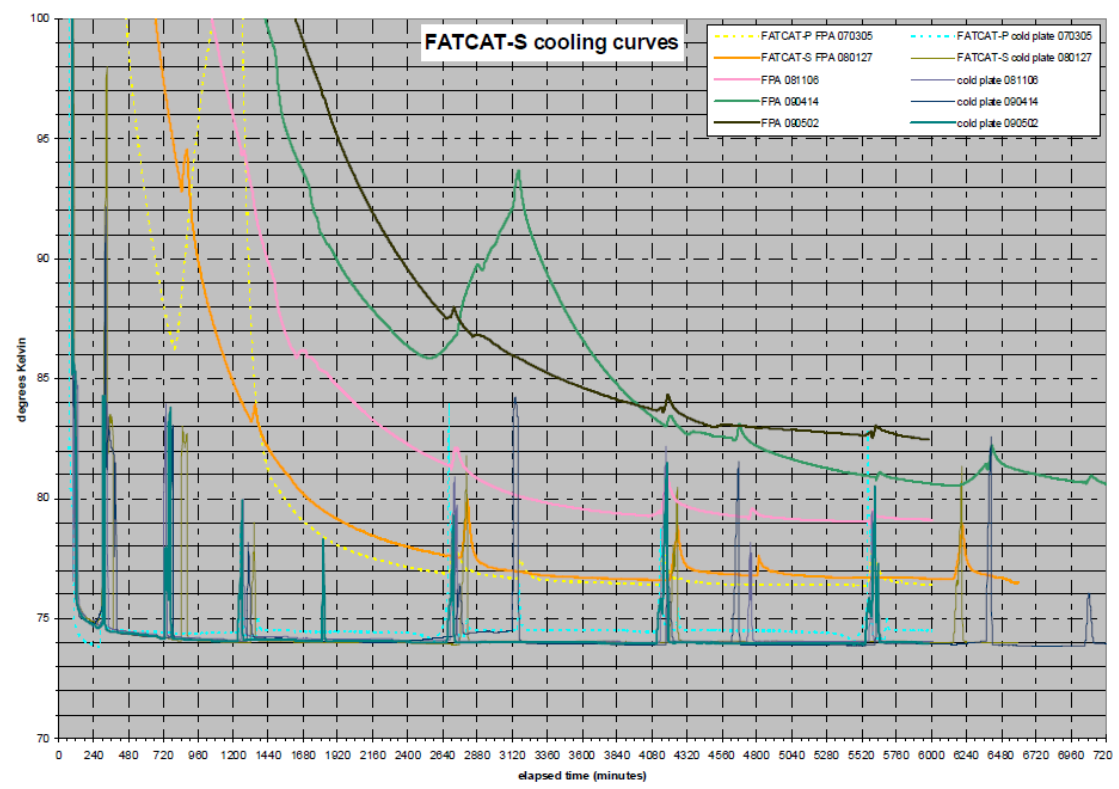

Figure 7: Degradation of the FATCAT-S FPA temperature over four thermal cycles from Jan 2008 through May 2009 (heavy orange, pink, green and black lines). For comparison, the dashed yellow line is from a March 2007 thermal cycle of the primary FATCAT. Nitrogen cold plate temperatures remain roughly constant over the period. Spikes in the graphs are present during liquid nitrogen fills.

During service missions to address other concerns, the instrument was carefully inspected for damage, thermal shorts to warm surfaces, and loose hardware. The vacuum and gas residuals were also carefully monitored. Tests were performed, both cold and at room temperature to rule out problems with the array. During a warm cycle, the mounting assembly for the array received special attention. The aluminum assembly is comprised of several pieces all screwed together. Figure 8 shows the bracket installed in the instrument with the FPA interface electronics and the cold strap.

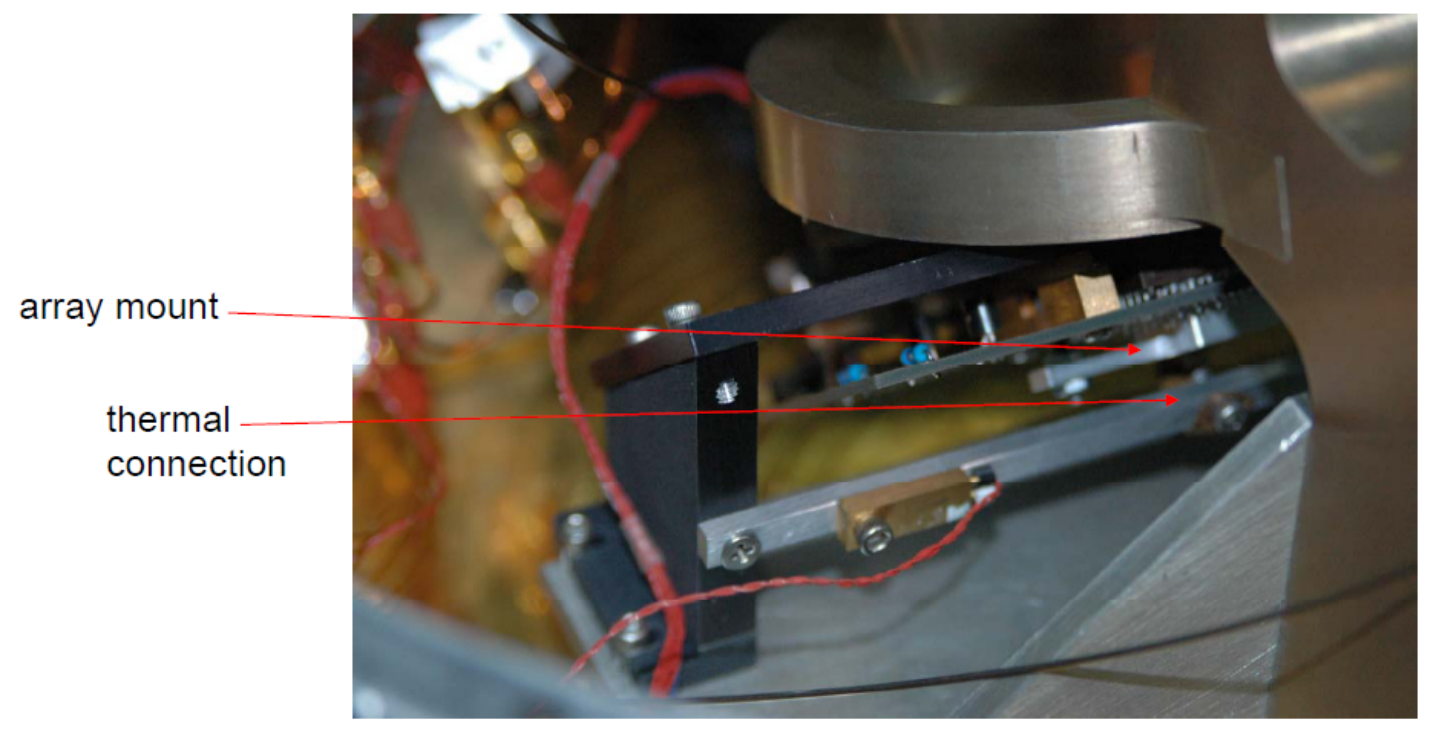

Figure 8: Array installed in instrument

Mechanical interfaces in the thermal path were lightly sanded to remove any oxidization and carefully cleaned. In a few places, the black anodization was removed to provide a better thermal path. The surfaces where the assembly mounts to 
the cold plate were flattened to ensure complete contact. All of the hardware was carefully tightened. Figure 9 identifies these interfaces.

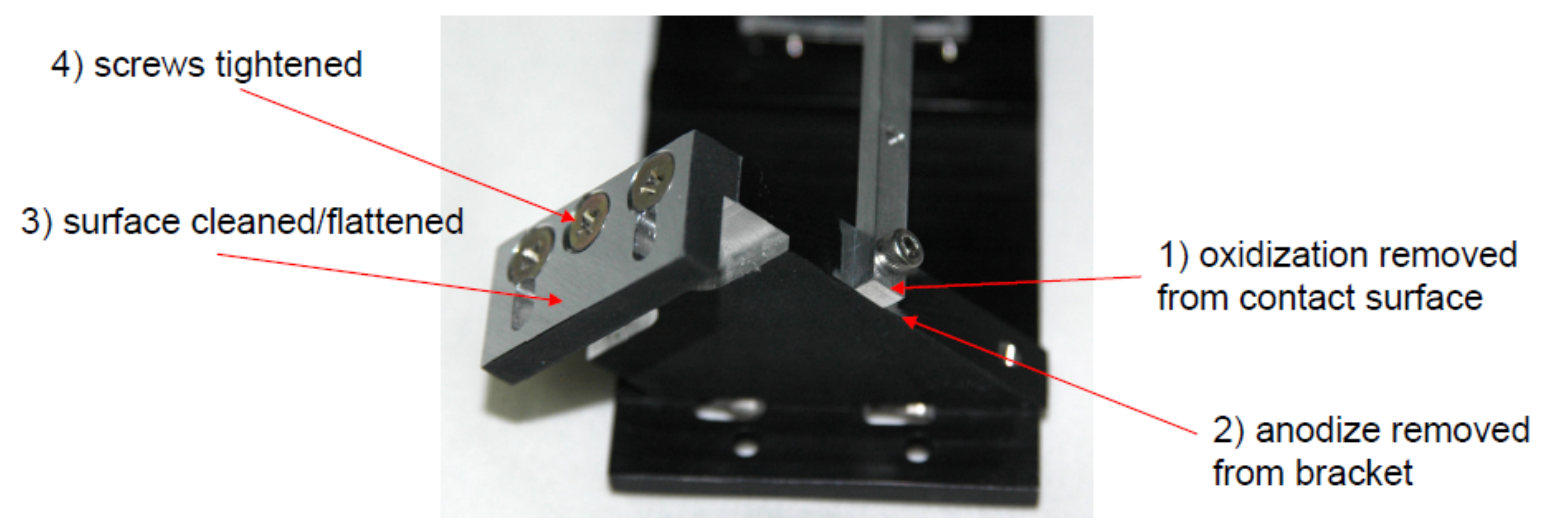

Figure 9: FATCAT-S Array Bracket. Several mechanical interfaces were improved to reduce the thermal impedance of the bracket assembly. Only one end of the bracket is shown. The array mounts just above the top of the figure.

After this work, the array cooled to its nominal temperature. The cool down was slightly faster than previous cycles with a cold plate temperature of $\sim 74 \mathrm{~K}$, as before. We conclude that numerous thermal cycles and the significant time that the instrument was open and exposed to atmosphere allowed hardware to loosen and the untreated aluminum to oxidize, resulting in degradation of the thermal path.

\subsection{Camera Motion Control Issues}

A long standing and elusive problem with the piezo stages used inside the secondary fringe tracker camera was finally identified and resolved. The fringe tracker has four ports that are fiber fed. Each port has a piezo-actuated stage used to position the beam on the detector. However, these had a poor range of motion at the cryogenic operating temperature. After several service missions on the instrument, it was finally determined that the piezo stack insulation was failing when cold. Now that specific cryo-rated piezo actuators have been installed, the problem is resolved.

The secondary fringe tracker also had a problem with its internal focus stage (Figure 10). A cryogenic stepper motor rotates a cam that applies force to a spring-loaded flexure that drives an optic mounted to the assembly. This was another case where proper functionality was not achieved at the $\sim 74 \mathrm{~K}$ operating temperature. The problem was traced to the cam follower (bearing) seizing at cold temperatures. We discovered that, after meticulous cleaning of the components, resurfacing of the cam and a slight increase in the operating current of the motor, the cam would slide against the seized bearing allowing adjustment of the focus. One of the lessons learned here was that the thin film of cryogenic grease applied to the cam and follower did more harm than good. Given the infrequent operation of this mechanism, it was decided that replacing the bearing with one that would function at cryogenic temperatures was not warranted. 


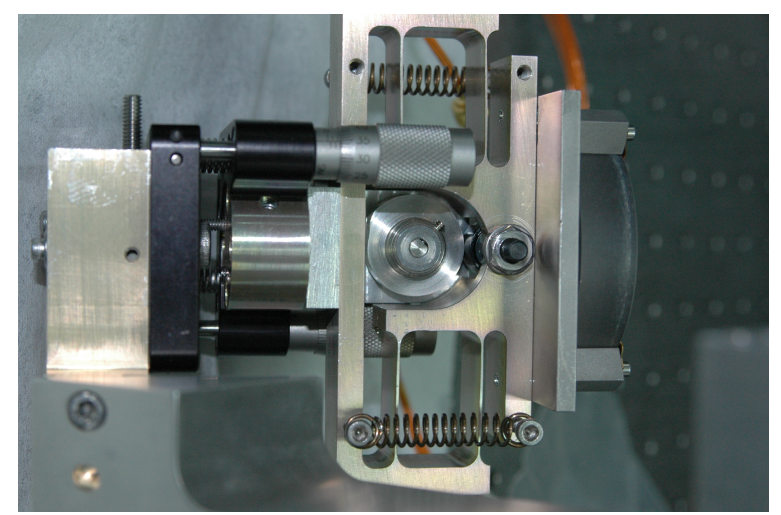

Figure 10: FATCAT-S camera focus mechanism. An eccentric cam (right of center) is rotated by a stepper motor (not visible). A bearing follower (black, to the right of the cam) provides a low friction interface with a springloaded flexure. A parabolic mirror (far right) is mounted to this flexure. The assembly provides $\sim 1.7 \mathrm{~mm}$ of translation needed to optimize focus on the IR detector. The micrometers are used for coarse focus and tip/tilt alignment.

\subsection{Pipeline Automation}

The KI now has a variety of modes of operation (V2, Nuller, and soon ASTRA) and possible configurations (spectral band, spectral resolution, slow integrations, and one or two data streams). In order to achieve more efficient operations with respect to the data pipelines, we have implemented new telemetry which carries information on the instrument mode and specific configuration at all times. This new telemetry is extracted by our near-real time and end-of-night processing pipelines, such that they are now able to run uninterrupted and without human intervention even when instrument reconfigurations take place during a given night. This automation is made possible by an underlying set of new (perl) scripts which generate a list of "events" requiring a change in the processing parameters, as well as the various configuration files required by the processing codes. These new scripts are also useful when used stand-alone in order to characterize specific pieces of data.

\section{SCIENCE OPERATIONS}

The visibility (V2) mode combines the adaptive optics corrected beams from the two Keck telescopes for measuring the fringe contrast in the near-infrared atmospheric windows. We had 28 observing runs since 1 July 2008 - 52 science nights (23 $\mathrm{V}^{2}$ Science \& 29 Nuller Science) and 14 nights for engineering (9.5 for $\mathrm{V}^{2}$ (including ASTRA engineering) \& 4.5 Nuller). There are nine refereed articles published from this instrument since the last 2008 SPIE meeting. The science results can be classified into four broad categories namely: (1) YSO disks, (2) pre-main sequence star masses, (3) active galactic nuclei, and (4) debris disks.

The nuller exozodiacal dust key science project was carried out between 2008 February and 2009 January (Keck semesters 2008A and 2008B). The overall project objective was to observe nearby main sequence stars that could be potential targets for future planet-finding missions, as well as to observe targets which were known to have debris disks. The project used 32 two-Keck nights over those two semesters, distributed as 28 full nights and 8 half nights, and arranged into 8 observing runs. Three key science teams, competitively selected in 2007 November, provided the targets. In total, of the 46 targets submitted by the three teams, 44 were observed as part of this key science project.

\section{FUTURE PLANS}

- ASTRA Project: Starting July 2006, WMKO was awarded a Major Research Instrumentation grant from the National Science Foundation to upgrade the existing interferometer through the ASTtrometry and phaseReferencing Astronomy (ASTRA) project ${ }^{8}$. The goal of this project is to develop three new modes: a Self Phase Referencing mode (on-axis phase referencing for high spectral resolution observations), a Dual Field Phase 
Referencing mode (off-axis phase referencing for fainter observations), and an Astrometric mode. The Self Phase Referencing mode is offered as a standard observing mode, , the Dual Field Phase Referencing is currently available to the community for shared-risk observations and the Astrometry mode is currently in development.

- Laser Guide Star (LGS) Implementation on Keck 1: The Keck 1 LGS development is not part of the KI project, but this capability would open-up the possibility of operating KI in laser guide star mode and hence observing highly obscured astronomical objects which are typically faint in the visible and bright in the infrared. Currently such observations are not possible, since only the Keck 2 telescope has LGS capability. The LGS capability for the Keck 1 telescope is expected to be complete by the end of 2010. The near-future K1 LGS and KI astrometry capabilities would enable addressing interesting astrophysical problems through galactic center observations ${ }^{15}$.

\section{ACKNOWLEDGMENTS}

The Keck Interferometer is funded by the National Aeronautics and Space Administration (NASA). Observations presented here were obtained at the W. M. Keck Observatory, which is operated as a scientific partnership among the California Institute of Technology, the University of California, and NASA. The Observatory was made possible by the generous financial support of the W. M. Keck Foundation. Part of this work was performed at the Jet Propulsion Laboratory, California Institute of Technology, and at the NASA Exoplanet Science Institute, California Institute of Technology, under contract with NASA.

\section{REFERENCES}

[1] Colavita, M. M., Wizinowich, P. L., \& Akeson, R. L., "Keck Interferometer status and plans," Proc. SPIE, 5491, 454 (2004).

[2] Wizinowich, P. L. et al., "Recent progress at the Keck Interferometer," Proc. SPIE, 6268, 62680N (2006).

[3] Colavita, M. M., Wizinowich, P. L., \& Akeson, R. L., "Nulling at the Keck Interferometer," Proc. SPIE, 6268, 626803 (2006).

[4] Ragland, S. D. et al., "Recent progress at the Keck Interferometer: operations and V2 science," Proc. SPIE, 7013, 70130B (2008).

[5] Colavita, M. M. et al., "Keck Interferometer nuller update," Proc. SPIE, 7013, 70130A (2008).

[6] Colavita, et al., "Keck Interferometer nuller instrument performance," Proc. SPIE, 7734, 7734-28 (2010). [this conference]

[7] Colavita, M. M. et al., "Keck Interferometer nuller data reduction and On-sky performance", PASP, 121, 1120 (2009).

[8] Woillez, J. M. et al., "ASTRA: the astrometric and phase-referencing astronomy upgrade for the Keck Interferometer," Proc. SPIE, 7734, 7734-37 (2010). [this conference]

[9] Akeson, R. L. et al., "Recent science highlights from the Keck Interferometer," Proc. SPIE, 7734, 7734-11 (2010). [this conference]

[10] Woillez, J. et al., in preparation (2010)

[11] Pott, J.-U. et al., Proc. SPIE, 7734, 7734-24 (2010). [this conference]

[12] Pott, J.-U. et al.., ApJ, submitted (2010)

[13] Eisner, J.A. et al., ApJ, submitted (2010)

[14] Ragland, S. et al.., ApJ, 703, 22 (2009)

[15]Eisner, J.A. et al., "Science with the Keck interferometer ASTRA program," Proc. SPIE, 7734, 7734-36 (2010). [this conference] 